\title{
Clinical application of indocyanine green fluorescence navigation technology to determine the safe margin of advanced oral squamous cell carcinoma
}

\author{
Zhuhao Wu ${ }^{1 \#}$, Yingchun Dong ${ }^{1 \#}$, Yuxin Wang ${ }^{1}$, Qingang Hu${ }^{1}$, Huiming Cai $^{2}$, Guowen Sun ${ }^{1}$ \\ ${ }^{1}$ Department of Maxillofacial Surgery, Nanjing Stomatological Hospital, Medical School of Nanjing University, Nanjing, China; ${ }^{2}$ Nanjing Nuoyuan \\ Medical Devices, Co., Ltd., Nanjing, China \\ Contributions: (I) Conception and design: G Sun; (II) Administrative support: Z Wu, Y Dong; (III) Provision of study materials or patients: G Sun; (IV) \\ Collection and assembly of data: Z Wu, Y Dong, H Cai; (V) Data analysis and interpretation: Y Wang, Q Hu; (VI) Manuscript writing: All authors; \\ (VII) Final approval of manuscript: All authors. \\ "These authors contributed equally to this work. \\ Correspondence to: Guowen Sun. Department of Maxillofacial Surgery, Nanjing Stomatological Hospital, Medical School of Nanjing University, \\ Nanjing, China. Email: 238957@sina.com.
}

\begin{abstract}
Background Advanced oral squamous cell carcinoma (OSCC) has large lesions and deep infiltration, and the control of safe surgical margins was difficult. If residual tumor remains after incomplete tumor resection, it can lead to local tumor recurrence or even distant metastasis. This study sought to investigate the clinical application of indocyanine green (ICG)-based near-infrared fluorescence (NIF) molecular imaging in the intraoperative detection of surgical margins of advanced OSCC.

Methods: Twenty-nine patients with advanced OSCC treated at the First Ward of Oral and Maxillofacial Surgery, Nanjing Stomatological Hospital were divided into the ICG group and non-ICG group. In the ICG group, the tumors were removed with the assistance of ICG fluorescence navigation technology. In the nonICG group, the tumors were removed with conventional methods, and the cutting-edge tissues of the two groups underwent frozen biopsies. The margin abnormality rates were calculated and compared.

Results: Under the excitation of NIF in the ICG group, tumor fluorescence development was observable in all lesions, and the tumor boundary was clear. The abnormal rates of the incisional margin in the ICG group and non-ICG group were $0.78 \%$ and $6.25 \%$, respectively $(\mathrm{P}<0.05)$.

Conclusions: ICG-mediated NIF imaging technology provides a new method for observing and completely resecting tumors under direct vision during operation, and finding residual tumors at the cutting edge in time. These results will inform the treatment of advanced OSCC.
\end{abstract}

Keywords: Near-infrared fluorescence molecular imaging technology; advanced oral squamous cell carcinoma (advanced OSCC); surgical safety margin; indocyanine green (ICG)

Submitted Dec 12, 2021. Accepted for publication Feb 16, 2022.

doi: $10.21037 /$ gs-22-33

View this article at: https://dx.doi.org/10.21037/gs-22-33

\section{Introduction}

Oral squamous cell carcinoma (OSCC) is a common epitheliogenic malignancy of the head and neck region (1). Clinically, a multidisciplinary treatment based on surgical treatment, supplemented by radiotherapy and chemotherapy, is often used. The large extent and deep invasion of advanced OSCC lesions make it difficult for physicians to judge the safe resection margin in the salvage surgery, especially the deep cutting edge in the surgical field. If residual tumor remains after incomplete tumor resection, it can lead to local tumor recurrence or even distant metastasis, and greatly reduce the survival rate of 
patients. The postoperative recurrence of OSCC due to incomplete surgical resection is one of the main causes of death of OSCC patients (2).

Near-infrared fluorescence (NIF) is a branch of optical molecular imaging that has the advantages of strong penetration, a low signal-to-noise ratio, and high sensitivity, and can assist in the intraoperative real-time imaging of tumors in complex anatomical structures, thereby guiding accurate tumor resection (3). At present, NIF technology has good prospects for use in tumor imaging, tumor localization, boundary determination, and tumor detection (4). Indocyanine green (ICG) is a cyanine fluorescent molecule, which emits fluorescence of near-infrared wavelength under the excitation of light of specific wavelength, and can be detected by NIF imaging equipment. ICG is characterized by its low trauma, low cytotoxicity, high time-effect, and high imaging resolution (5), and has been used in liver surgery (6), neurosurgery (7), and breast surgery (8). ICG has been used to conduct intraoperative searches and localize tumor satellite lesions and metastases (9). However, there are few reports on the application of ICG in advanced OSCC surgery. This study sought to examine the value of NIF navigation technology in the intraoperative and surgical margin determination of advanced oral cancer by evaluating the effect of intraoperative tumor fluorescence imaging in patients with advanced OSCC. We present the following article in accordance with the STROBE reporting checklist (available at https://gs.amegroups.com/article/ view/10.21037/gs-22-33/rc).

\section{Methods}

\section{Study methods}

A retrospective cohort study was conducted. According to the inclusion and exclusion criteria, 29 patients (21 males and 8 females) with advanced OSCC who received surgical treatment at the First Ward of Oral and Maxillofacial Surgery, Nanjing Stomatological Hospital from May 2020 to May 2021 were selected and divided into the ICG group $(n=13)$ and non-ICG group $(n=16)$ according to whether or not ICG fluorescence was used during surgery. The ICG group comprised 8 males and 5 females with an average age of 60.5 years. The non-ICG group comprised 13 males and 3 females with an average age of 58.1 years.

\section{Inclusion and exclusion criteria}

To be eligible for inclusion in the study, patients had to meet the following criteria (I) have been pathologically designed with OSCC, and have a T4 clinical stage tumor; (II) have a single tumor without distant metastasis; and (III) be aged 18-75 years old, and be male or female. Patients were excluded from the study if they met any of the following exclusion criteria: (I) had a history of maxillofacial chemoradiotherapy; (II) were allergic to ICG and iodine; and/or (III) had severe heart, lung, brain and kidney dysfunction, and could not tolerate surgery. All procedures performed in this study were in accordance with the Declaration of Helsinki (as revised in 2013). This experiment was approved by the Ethics Committee of Nanjing Stomatological Hospital (No. NJSH2021NL-061), and all patients were informed of the risks and signed the informed consent form.

\section{Surgical methods}

All the procedures in this study were performed by the same senior chief physician who had extensive experience in maxillofacial surgery. The method for the ICG group was as follows: (I) each patient received a peripheral intravenous injection of ICG at a dose of $0.75 \mathrm{mg} / \mathrm{kg} 10$ hours before surgery, and their vital signs were monitored. 10 hours later, an extended resection of the lesion and free flap transfer repair were performed under general anesthesia; (II) according to the requirements of the clinical trial protocol of "Fluorescence Image Navigation System", NIF imaging equipment (Nanjing Nuoyuan Medical Devices, Co., Ltd.) was used to detect NIF at the primary tumor site and surrounding surgical fields of the patient's tumor, and 3 types of images were obtained (i.e., fluorescence, fused, and color-scale images; Figure 1); (III) the location of the lesion was located with the aid of NIF to verify the surgical safe margin, and the resection margin was delineated at a safe range of 1.5-2.0 cm from the fluorescent margin of the primary tumor; (IV) real-time assisted guidance using ICG fluorescence during the resection of the tumor was used to determine the boundary between the tumor and the surrounding tissue. After the complete resection of the tumor tissue, NIF was again used to detect the tumor resection margin (Figure 2) and surgical bed fluorescence, and a further extended resection was performed if suspicious 

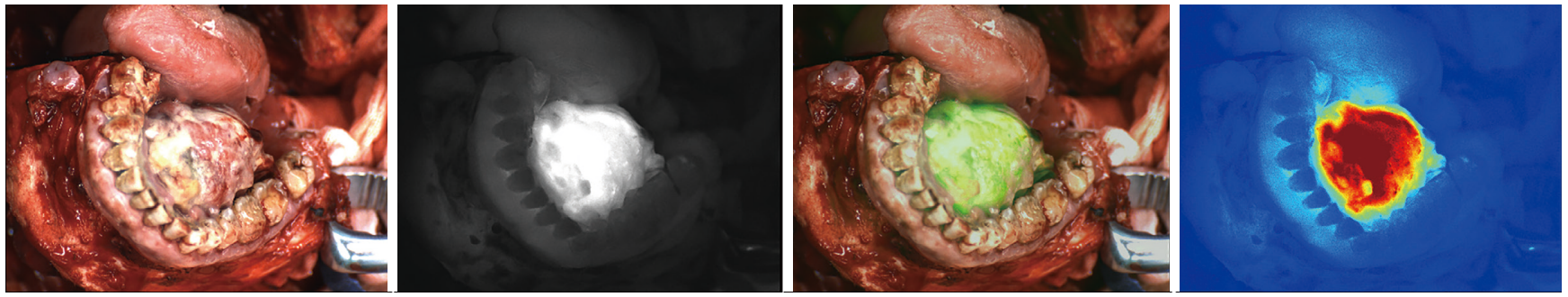

Figure 1 The primary tumor was detected by NIF with clear boundaries. NIF, near-infrared fluorescence.
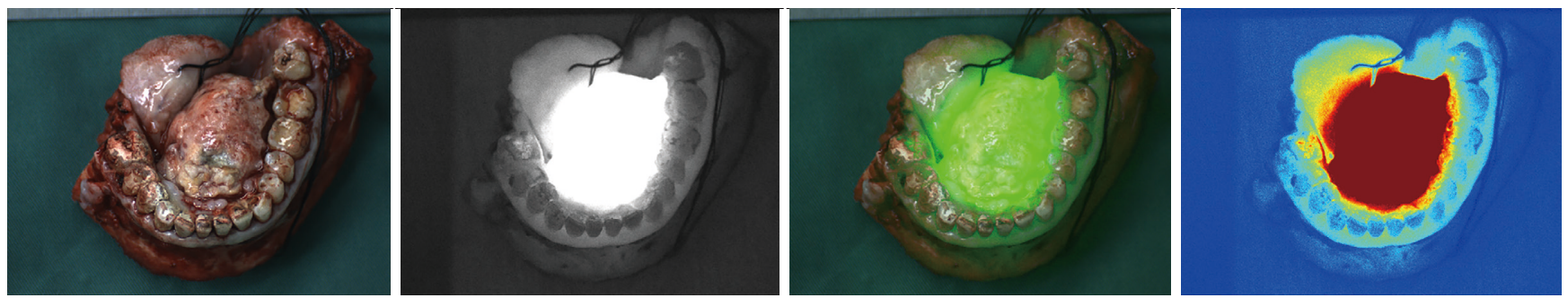

Figure 2 The isolated lesions were detected by NIF, and no abnormal fluorescence image was found at the cutting edge. NIF, near-infrared fluorescence.
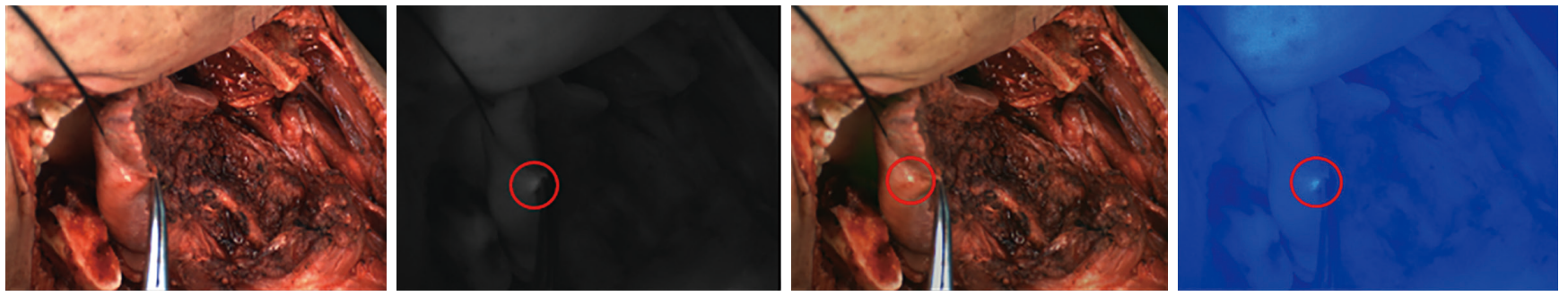

Figure 3 NIF detection was performed on the surgical field after the resection of the lesion. Abnormal fluorescence images were observed at the cutting edge. After a supplementary resection, an intraoperative frozen biopsy was performed. The results showed localized moderate to severe epithelial dysplasia. The red circle indicates the location of abnormal fluorescence imagines. NIF, near-infrared fluorescence.

fluorescence was observed (Figure 3); and (V) the resection margin was taken for the intraoperative rapid frozen biopsy, and if the resection margin was found to have residual cancer, real-time assisted guidance with ICG fluorescence was then used to enlarge the resection of the residual lesion under direct vision.

The method for the non-ICG group was as follows: (I) according to the preoperative clinical examination, the imaging data of the operation, and the clinical experience of the surgeon, a safe resection margin of the operation was determined based on a visual inspection and palpation; (II) the tumor was completely removed; (III) during the operation, the resection margin was taken from the location of the typical lesion of the tumor for an intraoperative rapid frozen biopsy, and if the resection margin was found to have residual cancer, a further extended resection was performed.

\section{Evaluation indicators}

The evaluation indicators were as follows: (I) the visualization of lesions in the ICG group by fluorescence, and a clear or unclear safe resection margin of surgery; (II) the abnormal resection margin rates of the ICG and the non-ICG groups, which were calculated according to the pathological diagnosis of the resection margin of the lesion using the following formula: the number of cases 
with abnormal resection margin by pathological diagnosis/ the number of cases with negative resection margin by pathological diagnosis + the number of cases with abnormal resection margin by pathological diagnosis; and (III) the detection of any residual tiny satellite lesions at the tumor resection margin and in the surgical bed during surgery by the ICG navigation technology.

\section{Statistical methods}

SPSS 18.0 software was used to analyze the abnormal rates of the resection margin of the ICG and non-ICG group by corrected chi-square tests, and the difference was considered statistically significant if the $\mathrm{P}$ value was $<0.05$.

\section{Results}

(I) ICG group: after the preoperative injection of ICG in 13 patients, tumor fluorescence was observed in the lesions under the excitation of NIF. The tumor boundary was clear, and its fluorescence boundary was located in the safe resection margin of surgery; (II) ICG group: there were 129 cases of resection margin tissue, and 1 case for which the pathology results revealed an abnormal resection margin; the abnormal resection margin was $0.78 \%$. Non-ICG group: there were 112 cases of resection margin tissue, and 7 cases with an abnormal resection margin; the abnormal resection margin was $6.25 \%$. The corrected chi-square test was used to confirm that there was a difference in the positive rates of the resection margin between the two groups $(\mathrm{P}<0.05)$; (III) ICG group: the lesions and surgical beds of 13 patients were observed with NIF. The surgical bed of 1 patient showed abnormal fluorescence lesions, and an intraoperative rapid frozen biopsy revealed localized moderate-severe abnormal hyperplasia of the epithelium. In 1 patient, the anterior resection margin of the lesion showed abnormal fluorescence images, and an intraoperative rapid frozen biopsy revealed inflammatory cell infiltration.

\section{Discussion}

At present, ICG fluorescence imaging technology has been used to assess the status of sentinel lymph nodes (10) and guide the resection of malignant tumors (11) in cancers, such as lung cancer, breast cancer, and liver cancer; however, ICG has been used less frequently in the resection of OSCC primary tumors. This study evaluated whether this technique has clinical application value in determining safe surgical margins during surgery for advanced OSCC.

In this study, $0.78 \%$ of the ICG group and $6.25 \%$ of the non-ICG group had abnormal margins. The corrected chisquare test showed that there was a difference in the rates of the abnormal margins between the two groups $(\mathrm{P}<0.05)$; that is, the use of ICG fluorescence navigation technology successfully reduced the rate of abnormal margins. Thus, the intraoperative application of this technique can help surgeons to more intuitively determine the tumor location, tumor boundary, and safe resection margin for surgery in real time, thereby reducing the abnormal rate of the resection margin.

At present, routine surgical safety margin determinations of advanced OSCC mainly rely on the following three methods: (I) an intraoperative visual inspection and palpation according to the clinical examination and imaging evaluation; (II) an intraoperative rapid frozen biopsy according to the personal experience of the surgeon; and (III) an intraoperative rapid frozen biopsy according to the sampling of the tumor margin or tissue around the tumor bed after tumor resection. However, advanced OSCC has a recurrence rate of $10-30 \%$ (12). This is because an intraoperative rapid frozen biopsy can only sample the tumor specimen and the typical lesion location of the tumor bed for pathological examination, and it is difficult to comprehensively assess the overall boundary of the tumor during surgery. In 1 case in the ICG group, the resection margin of the surgical bed showed abnormal fluorescence foci, and an intraoperative rapid frozen biopsy revealed localized moderate to severe dysplasia of the epithelium. In conventional surgery for advanced OSCC, it has great subjectivity that the operator usually selects several suspicious margins for the rapid frozen biopsy, and even if an intraoperative rapid frozen biopsy suggests residual cancer at the resection margin of the lesion, it is difficult for the surgeon to precisely determine the location of the abnormal resection margin in the surgical field. In this case, abnormal fluorescence was successfully detected in the surgical field using the ICG fluorescence navigation technique, and a supplementary resection was performed. This is because ICG fluorescence navigation technology can detect the whole operating table without omission, and conduct supplementary resection and examination of suspicious fluorescence points. Thus, the ICG fluorescence navigation technique can be used to assist in the resection of advanced OSCC, to determine the safe resection margin of the operation and reduce the local recurrence of the tumor, but further studies need to be conducted to confirm our findings. 
ICG is a near-infrared fluorescent dye certified by the United States Food and Drug Administration and the European Medicines Agency, its molecular weight is $751.4 \mathrm{Da}$ (13), with a half-life of 150 to $180 \mathrm{~s}$, and it can be metabolically cleared by the liver. ICG has an enhanced permeability and retention effect (14). By binding to hemoglobin in the macromolecules, ICG accumulates in vascular-rich tumor tissue (15), and when fluorescence is generated under near-infrared light excitation with wavelengths of 650 to $900 \mathrm{~nm}$, the tumor and its perimeter can be clearly visualized (16).

All 29 patients in this study had stage T4 advanced OSCC with a large extent of lesions and deep invasion, and the control of safe surgical margins was difficult, which can easily cause incomplete or excessive tumor resection (17). In 2013, Yokoyama et al. (18), observed 5 patients with oral cancer using ICG NIF imaging technology for the first time, and found that the sensitivity of ICG NIF imaging for head and neck squamous cell carcinoma was $100 \%$. Subsequent studies have also confirmed that ICG is a good molecular tracer for OSCC (19). In this study, we imaged the tumor lesions using the ICG fluorescence navigation technique for all 13 patients in the ICG group, and had a positive rate of $100 \%$. ICG fluorescent tumor borders can be clearly visualized and the completeness of intraoperative detection of tumor resection can be achieved by imaging equipment.

Notably, 1 patient had an abnormal fluorescent image at the anterior resection margin of the lesion, which was complemented by the surgeon and sent for an intraoperative rapid frozen biopsy, which confirmed inflammatory cell infiltration rather than residual cancer cells, which was associated with a false-positive reaction to ICG. ICG can accumulate in tumors or be enriched in normal glands, dense tissue, gingiva, or inflammatory tissue. The limitations of ICG fluorescence technology have not yet been solved. The false negative reaction of ICG refers to a situation in which ICG fluorescence is negative, and there is a pathological diagnosis of an abnormal resection margin; however, no false negative cases of ICG appear to have been reported at home and abroad. One patient developed ICG allergy in this clinical study, manifested as continuous low fever, and the other patients did not have adverse reactions.

When the ICG fluorescence technique is used for detection, intraoperative gray image has high practicability. Gray value measurement converts the abstract brightness contrast into specific contrast data. These data enable surgeons to quickly evaluate the visual fluorescence of the whole organ surface and help surgeons to make rapid judgments about the intraoperative resection margin. This technique has successfully been used in the intraoperative real-time dynamic assessments of tumor borders. It solves the problem related to safe resection margins that are difficult to locate in advanced OSCC surgery. On the basis of gray values, we can determine the sensitivity and specificity of NIF in distinguishing between normal margins and abnormal margins at the fluorescence boundary by calculating the tumor to background ratio value (i.e., the mean gray value of tumor tissue/the gray value of surrounding tissue). Thus, this is a new feasible method for discerning potentially malignant lesion tissues.

In summary, ICG fluorescence imaging technology provides a new approach for guiding the safe margin determination and complete resection of salvage surgery for advanced OSCC involving important anatomical structures and with deep margins, and has promising clinical applications.

\section{Acknowledgments}

Funding: The study was supported by accurate repair of jaw deformity assisted by navigation technology (No. 201503038).

\section{Footnote}

Reporting Checklist: The authors have completed the STROBE reporting checklist. Available at https:// gs.amegroups.com/article/view/10.21037/gs-22-33/rc

Data Sharing Statement: Available at https://gs.amegroups. com/article/view/10.21037/gs-22-33/dss

Conflicts of Interest: All authors have completed the ICMJE uniform disclosure form (available at https://gs.amegroups. com/article/view/10.21037/gs-22-33/coif). HC is from Nanjing Nuoyuan Medical Devices, Co., Ltd. The other authors have no conflicts of interest to declare.

Ethical Statement: The authors are accountable for all aspects of the work in ensuring that questions related to the accuracy or integrity of any part of the work are appropriately investigated and resolved. All procedures performed in this study were in accordance with the Declaration of Helsinki (as revised in 2013). This experiment was approved by the Ethics Committee of Nanjing Stomatological Hospital (No. NJSH- 
2021NL-061), and all patients were informed of the risks and signed the informed consent form.

Open Access Statement: This is an Open Access article distributed in accordance with the Creative Commons Attribution-NonCommercial-NoDerivs 4.0 International License (CC BY-NC-ND 4.0), which permits the noncommercial replication and distribution of the article with the strict proviso that no changes or edits are made and the original work is properly cited (including links to both the formal publication through the relevant DOI and the license). See: https://creativecommons.org/licenses/by-nc-nd/4.0/.

\section{References}

1. Sawair FA, Irwin CR, Gordon DJ, et al. Invasive front grading: reliability and usefulness in the management of oral squamous cell carcinoma. J Oral Pathol Med 2003;32:1-9.

2. Cristaldi M, Mauceri R, Di Fede O, et al. Salivary Biomarkers for Oral Squamous Cell Carcinoma Diagnosis and Follow-Up: Current Status and Perspectives. Front Physiol 2019;10:1476.

3. DSouza AV, Lin H, Henderson ER, et al. Review of fluorescence guided surgery systems: identification of key performance capabilities beyond indocyanine green imaging. J Biomed Opt 2016;21:80901.

4. Spartalis E, Ntokos G, Georgiou K, et al. Intraoperative Indocyanine Green (ICG) Angiography for the Identification of the Parathyroid Glands: Current Evidence and Future Perspectives. In Vivo 2020;34:23-32.

5. Lau CT, Au DM, Wong KKY. Application of indocyanine green in pediatric surgery. Pediatr Surg Int 2019;35:1035-41.

6. Wang X, Teh CSC, Ishizawa T, et al. Consensus Guidelines for the Use of Fluorescence Imaging in Hepatobiliary Surgery. Ann Surg 2021;274:97-106.

7. Chatterjee A, Krishnan NM, Van Vliet MM, et al. A comparison of free autologous breast reconstruction with and without the use of laser-assisted indocyanine green angiography: a cost-effectiveness analysis. Plast Reconstr Surg 2013;131:693e-701e.

8. Jin $\mathrm{X}, \mathrm{Lu} \mathrm{X}$, Zhang $\mathrm{Z}$, et al. Indocyanine GreenParthenolide Thermosensitive Liposome Combination Treatment for Triple-Negative Breast Cancer. Int J Nanomedicine 2020;15:3193-206.

9. Mandovra P, Kalikar V, Patankar RV. Real-Time Visualization of Ureters Using Indocyanine Green During Laparoscopic Surgeries: Can We Make Surgery Safer?
Surg Innov 2019;26:464-8.

10. Kim JH, Ku M, Yang J, et al. Recent Developments of ICG-Guided Sentinel Lymph Node Mapping in Oral Cancer. Diagnostics (Basel) 2021;11:891.

11. Liberale G, Bourgeois P, Larsimont D, et al. Indocyanine green fluorescence-guided surgery after IV injection in metastatic colorectal cancer: A systematic review. Eur J Surg Oncol 2017;43:1656-67.

12. Liao CT, Chang JT, Wang HM, et al. Analysis of risk factors of predictive local tumor control in oral cavity cancer. Ann Surg Oncol 2008;15:915-22.

13. Reinhart MB, Huntington CR, Blair LJ, et al. Indocyanine Green: Historical Context, Current Applications, and Future Considerations. Surg Innov 2016;23:166-75.

14. Maeda H. Toward a full understanding of the EPR effect in primary and metastatic tumors as well as issues related to its heterogeneity. Adv Drug Deliv Rev 2015;91:3-6.

15. Maeda $\mathrm{H}$. The enhanced permeability and retention (EPR) effect in tumor vasculature: the key role of tumor-selective macromolecular drug targeting. Adv Enzyme Regul 2001;41:189-207.

16. Wang Y, Xie D, Pan J, et al. A near infrared lighttriggered human serum albumin drug delivery system with coordination bonding of indocyanine green and cisplatin for targeting photochemistry therapy against oral squamous cell cancer. Biomater Sci 2019;7:5270-82.

17. Krall AS, Xu S, Graeber TG, et al. Asparagine promotes cancer cell proliferation through use as an amino acid exchange factor. Nat Commun 2016;7:11457.

18. Yokoyama J, Fujimaki M, Ohba S, et al. A feasibility study of NIR fluorescent image-guided surgery in head and neck cancer based on the assessment of optimum surgical time as revealed through dynamic imaging. Onco Targets Ther 2013;6:325-30.

19. Al-Dam A, Precht C, Barbe A, et al. Sensitivity and specificity of sentinel lymph node biopsy in patients with oral squamous cell carcinomas using indocyanine green fluorescence imaging. J Craniomaxillofac Surg 2018;46:1379-84.

(English Language Editor: L. Huleatt)

Cite this article as: Wu Z, Dong Y, Wang Y, Hu Q, Cai H, Sun G. Clinical application of indocyanine green fluorescence navigation technology to determine the safe margin of advanced oral squamous cell carcinoma. Gland Surg 2022;11(2):352-357. doi: 10.21037/gs-22-33 